\title{
Finite-Time Asynchronous Stabilization for Nonlinear Hidden Markov Jump Systems with Parameter Varying in Continuous-Time Case
}

\author{
Lianjun Xiao $\mathbb{D}^{1},{ }^{1}$ Xiaofeng Wang $\mathbb{D}^{2},{ }^{2}$ and Lingling Gao $\mathbb{D}^{1}$ \\ ${ }^{1}$ School of Artificial Intelligence and Big Data, Hefei University, Hefei 230601, China \\ ${ }^{2}$ Anhui Provincial Engineering Laboratory of Big Data Technology Application for Urban Infrastructure, \\ School of Artificial Intelligence and Big Data, Hefei University, Hefei 230601, China \\ Correspondence should be addressed to Xiaofeng Wang; xfwang@hfuu.edu.cn
}

Received 12 July 2020; Revised 2 September 2020; Accepted 25 September 2020; Published 28 October 2020

Academic Editor: Shuping He

Copyright (C) 2020 Lianjun Xiao et al. This is an open access article distributed under the Creative Commons Attribution License, which permits unrestricted use, distribution, and reproduction in any medium, provided the original work is properly cited.

The finite-time asynchronous stabilization problem has received great attention because of the wide application of actual engineering. In this paper, we consider the problem of finite-time asynchronous stabilization for nonlinear hidden Markov jump systems (HMJSs) with linear parameter varying. Compared with the existing research results on Markov jump systems, this paper considers the HMJSs which contain both the hidden state and the observed state in continuous-time case. Moreover, we consider the parameters of the systems are time varying. The aim of the paper is to design a proper observation-mode-based asynchronous controller such that the closed-loop HMJSs with linear parameter varying be stochastically finite-time bounded with $H_{\infty}$ performance (SFTB- $H_{\infty}$ ). Then, we give some sufficient conditions to solve the SFTB- $H_{\infty}$ asynchronous controller by considering the stochastic Lyapunov-Krasovskii functional (SLKF) methods. Finally, a numerical example is used to show the validity of the main results.

\section{Introduction}

In recent years, hybrid systems have attracted the great attention and research due to their wide application in the industrial control field. Moreover, with the continuous upgrading and changes in the industrial environment, how to model and control hybrid systems in complex environments has become a major research hotspot in the control field. As a kind of special hybrid systems, Markov jump systems (MJSs) have received researchers' great attention and many constructive results have been made. It should be noted that the synchronous controllers are always considered in many existing results when it comes to the stabilization problem of Markov jump systems. That means the controller of the systems can always track the information of the model of the system in real time. However, such a situation is always impossible to achieve in a Markov jump system with a complex environment. Therefore, the asynchronous characteristic between the system modal and the controller has attracted the attention of many researchers and they began to focus on the various characteristics of the HMJSs. The static output constrained control [1], the output feedback control [2], and the observer-based asynchronous fault detection [3] problems of the HMJSs with the discretetime state are studied by the authors, respectively. In [4], the resilient asynchronous $H_{\infty}$ control problem of the discretetime HMJSs with singularly perturbed was considered. For the nonlinear HMJSs, the quantized control [5] and the finite-time $L_{2}$-gain asynchronous control [6] problems by using T-S fuzzy model approach are studied, respectively. Moreover, many researchers also have carried out some work for the continuous-time HMJSs. The asynchronous $\mathrm{H}_{2}$-controller design and the asynchronous filter design problems are studied for the continuous-time HMJSs in $[7,8]$, respectively. For more particulars of HMJSs, the interested readers can read references $[9,10]$.

It should be pointed out that many results of the MJSs assume that the parameter matrices are constant matrices or 
uncertain matrices that satisfy some given known conditions. In actual control systems, the system parameters may have the characteristics of convex polygonal linear parameters varying due to the sensor and actuator failures. For the control systems with such a special situation, it is necessary to design the corresponding nonlinear parameter timevarying controller to stabilization of the systems. Thus, many researchers proposed the linear parameter-varying control methods which laid a solid theoretical foundation for the design of nonlinear parameter time-varying controller. For a kind of parameter-varying system [11], an adaptive algorithm was proposed to achieve the active vibration control by using a new online secondary path estimation method. For bounded parameter variation linear parameter-varying systems, the LMI-based filter design problem is studied in [12]. The robust fault estimation [13] and set-membership fault estimation [14] problem is studied for parameter timevarying systems. For more particulars of parameter-varying systems, the interested readers can read references [15-18].

In the abovementioned references, the system analysis and/or some control method were concerned only over an infinite-time interval, which portrayed the asymptotic properties of the HMJSs and parameter-varying systems. However, the transient characteristics in a given finite-time interval is significant in many control systems [19-21] and should be considered simultaneously. For a class of nonlinear systems, the authors studied the finite-time adaptive fuzzy control problem in [22, 23], respectively. In [24], a finite-time control approach was used to solve the problem of accurate trajectory tracking for disturbed surface vehicles. Moreover, many researchers also combine the finite-time control scheme with the networked switched systems [25], nonlinear systems [26, 27], and quadrotors control [28] and have carried out lots of excellent works. The authors studied the design and implementation of bounded finite-time control algorithm problem for the speed regulation of permanent magnet synchronous motor in [29]. By using the state-dependent switching method, the adaptive fuzzy finitetime control of switched nonlinear systems is studied in [30]. The problem of fuzzy finite-time control for switched systems via adding a barrier power integrator is considered in [31]. For more particulars of HMJSs, the interested readers can read references [32-34].

However, few results have been reported regarding research on finite-time asynchronous control of HMJSs based on the parameter varying, which is the motivation of this work. Different from some existing results on the finite-time control problem $[35,36]$, the problem of SFTB- $H_{\infty}$ asynchronous control is studied for continuous-time HMJSs via parameter varying in this paper. Compared with the existing results of asynchronous control for discrete-time HMJSs [37-40], this paper mainly consists of the following threefold contributions:

(1) The asynchronous characteristic between the controller modes and the system modes is characterized by the hidden Markov dynamics. Moreover, we firstly consider the asynchronous stabilization problem for the continuous-time HMJSs with parameter varying models.

(2) Some sufficient conditions will be given to solve the SFTB- $H_{\infty}$ observation-mode-based asynchronous controller by considering the SLKF methods and introducing some auxiliary variables.

(3) Considering the parameter time-varying, the methods of gridding technique and approximate basis function will be used in order to change the infinite LMIs into finite LMIs which can be solved by MATLAB LMI toolbox to get the finite-time asynchronous controller gain directly.

The organization of this paper is made of five parts. In Section 1, the background of the HMJSs, the parameter timevarying systems, finite-time control scheme, and the notation meaning of this paper are introduced and given. Section 2 introduces the system description of the parameter varying hidden Markov jump systems (PV-HMJSs) and designs a suitable asynchronous controller for the studied systems. Moreover, the main definitions and lemmas are also given in this part. Section 3 gives some sufficient conditions to solve the SFTB- $H_{\infty}$ observation-mode-based asynchronous controller. In Section 4, the simulation experiment of HMJSs via parameter varying with two subsystems is carried out, only to find that the closed-loop HMJSs via parameter varying fulfill the condition of SFTB- $H_{\infty}$ under the action of the designed asynchronous controller. The conclusion and future research work follow in Section 5.

Notation: the notation meaning throughout this paper is shown in Table 1. Furthermore, we assume that the matrices and notations in this paper are standard with compossible dimensions.

\section{System Formulation}

2.1. System Description. We consider the nonlinear parameter varying hidden Markov jump systems (PV-HMJSs) defined on the probability space $(\Gamma, \Delta, \operatorname{Prob}(\cdot))$ :

$$
\left\{\begin{array}{l}
{\left[\begin{array}{l}
\dot{x}(t) \\
y(t)
\end{array}\right]=f_{H(t)}^{p(t)}(x(t), \omega(t))+g_{H(t)}^{p(t)} u(t)} \\
x(t)=x_{0} \\
p(t)=p_{0} \\
H(t)=H_{0} \\
t=0
\end{array}\right.
$$

where $H(t)=\left(h_{1}(t), h_{2}(t)\right)$ and $p(t)=\left[p_{1}(t), p_{2}(t)\right.$, $\left.\ldots, p_{m}(t)\right]^{\mathrm{T}}$. The notations of the PV-HMJSs (1) are given in Table 2.

The right-continuous hidden Markov chain $H(t)$ is composed of the hidden state $h_{1}(t)$ in the finite set $\mathscr{M}:=$ $\{1,2, \ldots, M\}$ and the observation state $h_{2}(t)$ in the finite set $\mathscr{M}:=\{1,2, \ldots, N\}$. Furthermore, the hidden Markov chain $H(t) \in \mathscr{M} \times \mathscr{M}$ can be seen as a homogeneous Markov process which satisfies 
TABLE 1: Symbol notations.

\begin{tabular}{lc}
\hline Notation & Denotes \\
\hline $\mathbb{R}^{n}$ & $n$-dimensional Euclidean space \\
$\mathbb{R}^{n \times m}$ & $n \times m$ real matrices \\
$*$ & symmetric matrix \\
$\Im V$ & The weak infinitesimal operator of $V$ \\
$I$ & Unit matrix \\
$A^{\mathrm{T}}$ & Matrix transpose \\
$A^{-1}$ & Matrix inverse \\
0 & Zero matrix \\
$\|*\|$ & Euclidean vector norm \\
$\operatorname{Her}(A)$ & $A+A^{\mathrm{T}}$ \\
$\lambda_{\max }(P)$ & Maximum eigenvalue of $P$ \\
$\lambda_{\min }(P)$ & Minimum eigenvalue of $P$ \\
$\operatorname{diag}\{A B\}$ & The block-diagonal matrix of $A$ and $B$ \\
$\Gamma$ & The sample space \\
$\Delta$ & The algebra of events \\
$\mathbb{P}(\cdot)$ & The probability measure which defined on $\Delta$ \\
\hline
\end{tabular}

$$
\mathbb{P}(H(t+\tau)=(\mathscr{F}, \mathscr{L}) \mid H(t)=(\mathscr{I}, \mathscr{K}))= \begin{cases}\mathscr{R}_{(\mathscr{I}, \mathscr{K}),(\mathscr{F}, \mathscr{L})} \tau+o(\tau), & (\mathscr{I}, \mathscr{K}) \neq(\mathscr{J}, \mathscr{L}), \\ 1+\mathscr{R}_{(\mathscr{F}, \mathscr{K}),(\mathscr{I}, \mathscr{L})} \tau+o(\tau), & (\mathscr{I}, \mathscr{K})=(\mathscr{J}, \mathscr{L}),\end{cases}
$$

where $\mathscr{R}_{(\mathscr{T}, \mathscr{K}),(\mathscr{F}, \mathscr{L})} \geq 0, \forall(\mathscr{I}, \mathscr{K}) \neq(\mathscr{J}, \mathscr{L})$ is the transition rate with $\sum_{(\mathscr{F}, \mathscr{K}) \neq(\mathscr{f}, \mathscr{L})} \mathscr{R}_{(\mathscr{F}, \mathscr{K}),(\mathscr{F} \mathscr{L})}=-r_{(\mathscr{F}, \mathscr{K})}$ and for any $\mathscr{L}, \mathscr{K} \in \mathscr{M}, \mathscr{I}, \mathscr{J} \in \mathscr{M}$, which satisfies

$$
\mathscr{R}_{(\mathscr{F}, \mathscr{K}),(\mathscr{F}, \mathscr{L})}= \begin{cases}\alpha_{\mathscr{F} \mathscr{L}}^{\mathscr{K}} \pi_{\mathscr{I} \mathscr{I}}, & \mathscr{I} \neq \mathscr{I}, \\ q_{\mathscr{K} \mathscr{L}}^{\mathscr{I}}, & \mathscr{L} \neq \mathscr{K}, \mathscr{J}=\mathscr{I}, \\ \pi_{\mathscr{F} \mathscr{f}}+q_{\mathscr{K} \mathscr{K}}^{\mathscr{I}}, & \mathscr{J}=\mathscr{I}, \mathscr{K}=\mathscr{L} \\ 0, & \text { otherwise, }\end{cases}
$$

in which $\sum_{\mathscr{L}=1}^{N} \alpha_{\mathscr{F} \mathscr{L}}^{\mathscr{K}}=1, \Pi=\left[\pi_{\mathscr{F} \mathscr{f}}\right]$ is the transition rate matrix and satisfies $\pi_{\mathscr{I} \mathscr{F}} \geq 0$ for $\forall \mathscr{I} \neq \mathscr{J}, \aleph^{\mathscr{I}}=\left[q_{\mathscr{K} \mathscr{L}}^{\mathscr{I}}\right]$ is the $\Pi$-dependent conditional probability matrix and satisfies $q_{\mathscr{K} \mathscr{L}}^{\mathscr{T}} \geq 0 \quad$ for $\quad \forall \mathscr{K} \neq \mathscr{L}, \quad \pi_{\mathscr{I} \mathscr{I}}=-\sum_{\mathscr{I} \neq \mathscr{F}} \pi_{\mathscr{I} \mathscr{F}}, \quad$ and $q_{\mathscr{K} \mathscr{K}}=-\sum_{\mathscr{L} \neq \mathscr{K}} q_{\mathscr{K} \mathscr{L}}^{\mathscr{S}}$.

Remark 1. From relation (3), we can summarize that the same state will be visited of the hidden state $h_{1}(t)$ and the observation state $h_{2}(t)$ if $\mathscr{M}=\mathscr{M}, \alpha_{\mathscr{F} \mathscr{L}}^{\mathscr{L}}=0$ with $\mathscr{J} \neq \mathscr{L}$, and $\alpha_{\mathscr{F}}^{\mathscr{K}}=1$ with $\mathscr{J}=\mathscr{L}$. In this case, the full information of the hidden state $h_{1}(t)$ will be provided for the detector. If $\alpha_{\mathscr{Y} \mathscr{L}}^{\mathscr{K}}=$ 1 and $\alpha_{\mathscr{L} \mathscr{L}}^{\mathscr{L}}=0$ with $\mathscr{K} \neq \mathscr{L}$, only one jump will occur between the hidden state $h_{1}(t)$ and the observation state $h_{2}(t)$. Moreover, the observer will not provide any information if $\mathscr{M}=\{1\}, q_{\mathscr{K} \mathscr{L}}^{\mathscr{J}}=0$, and $\alpha_{\mathscr{I} 1}^{1}=1$ and the observation state is $h_{2}(t)=1$. For more specific details of the relationship between the hidden state $h_{1}(t)$ and the observation state $h_{2}(t)$, we can refer to $[3,6,35,36]$.

In actual engineering applications, we often encounter situations where the system mode information accessible by the controller/observer is usually inaccurate. In other words, the actual model of the system cannot be observed by the controller, which makes the information between the controller mode and the system mode asynchronous. Therefore, a new controller mode $h_{2}(t)$ related to the system mode $h_{1}(t)$ needs to be introduced.

The PV-HMJSs (1) can be rewritten as the following PVHMJSs:

$$
\left\{\begin{array}{l}
{\left[\begin{array}{l}
\dot{x}(t) \\
y(t)
\end{array}\right]=\mathbf{F}_{h_{1}(t)}^{p(t)} \mathbf{G}[x(t), u(t), \omega(t)]} \\
x(t)=x_{0} \\
p(t)=p_{0} \\
h_{1}(t)=h_{10} \\
t=0 \\
h_{1}(t) \in \mathscr{M}
\end{array}\right.
$$

where

$$
\mathbf{F}_{h_{1}(t)}^{p(t)}=\left[\begin{array}{cccc}
A_{h_{1}(t)}^{p(t)} & B_{h_{1}(t)}^{p(t)} & W_{1 h_{1}(t)}^{p(t)} & F_{h_{1}(t)}^{p(t)} \\
C_{h_{1}(t)}^{p(t)} & D_{h_{1}(t)}^{p(t)} & W_{2 h_{1}(t)}^{p(t)} & 0
\end{array}\right] .
$$

$$
\mathbf{G}[x(t), u(t), \omega(t)]=\operatorname{col}[x(t), u(t), \omega(t), f(x(t), t)],
$$

$A_{H(t)}^{p(t)}$ is the time-varying parameter-depende nt matrix, and $f(x(t), t)$ is the unknown nonlinear function.

We use $A_{\mathscr{I}}^{p(t)}, B_{\mathscr{I}}^{p(t)}, W_{1 \mathscr{J}}^{p(t)}, F_{\mathscr{I}}^{p(t)}, C_{\mathscr{I}}^{p(t)}, D_{\mathscr{I}}^{p(t)}$, and $W_{2 \mathscr{I}}^{p(t)}$ to denote $A_{h_{1}(t)}^{p(t)}, B_{h_{1}(t)}^{p(t)}, W_{1 h_{1}(t)}^{p(t)}, F_{h_{1}(t)}^{p(t)}, C_{h_{1}(t)}^{p(t)}, D_{h_{1}(t)}^{p(t)}$, and $W_{2 h_{1}(t)}^{p(t)}$, when $h_{1}(t)=\mathscr{F}, \mathscr{I} \in \mathscr{M}$, respectively.

For any $n=1,2, \ldots, m$, the time-varying parameter matrix $p(t)$ and its variation rate $\dot{p}(t)$ are both assumed bounded, i.e., $p_{n}(t) \in\left[\begin{array}{ll}\underline{p}_{1} & \bar{p}_{1}\end{array}\right]$ and $\dot{p}_{n}(t) \in\left[\begin{array}{ll}\underline{p}_{2} & \bar{p}_{2}\end{array}\right]$. Moreover, the time-varying parameter matrix $p(t)$ is also measurable and affine parameter dependent in real time. That means the following equations hold: 
TABle 2: The notations of the PV-HMJSs (1).

\begin{tabular}{lc}
\hline Notation & Denotes \\
\hline$x(t) \in \mathbb{R}^{n}$ & The systems state \\
$y(t) \in \mathbb{R}^{m}$ & The controlled output \\
$u(t) \in \mathbb{R}^{p}$ & The controlled input \\
$\omega(t) \in \mathbb{R}^{q}$ & The disturbance input \\
$p(t)$ & The time-varying parameter matrix \\
$H(t)$ & A right-continuous hidden Markov chain \\
$x_{0}$ & The initial state of the systems \\
$p_{0}$ & The initial time-varying parameter matrix \\
$H_{0}$ & The initial hidden Markov process \\
\hline
\end{tabular}

$$
\begin{aligned}
A_{\mathscr{I}}^{p} & =A_{0}+\sum_{\mathscr{I}=1}^{m} A_{\mathscr{I}} P_{\mathscr{I}}, \\
B_{\mathscr{I}}^{p} & =B_{0}+\sum_{\mathscr{I}=1}^{m} B_{\mathscr{I}} P_{\mathscr{I}}, \\
W_{1 \mathscr{I}}^{p} & =W_{10}+\sum_{\mathscr{I}=1}^{m} W_{1 \mathscr{I}} P_{\mathscr{I}}, \\
C_{\mathscr{I}}^{p} & =C_{0}+\sum_{\mathscr{I}=1}^{m} C_{\mathscr{I}} P_{\mathscr{I}}, \\
D_{\mathscr{I}}^{p} & =D_{0}+\sum_{\mathscr{I}=1}^{m} D_{\mathscr{I}} P_{\mathscr{I}}, \\
W_{2 \mathscr{I}}^{p} & =W_{20}+\sum_{\mathscr{I}=1}^{m} W_{2 \mathscr{I}} P_{\mathscr{I}}, \\
F \mathscr{I} P & =F_{0}+\sum_{\mathscr{I}=1}^{m} F_{\mathscr{I}} P_{\mathscr{I}},
\end{aligned}
$$

where $A_{\mathscr{I}}, B_{\mathscr{F}}, W_{1 \mathscr{I}}, F_{\mathscr{I}}, C_{\mathscr{I}}, D_{\mathscr{I}}$, and $W_{2 \mathscr{I}}$ with $\mathscr{I}=$ $0,1, \ldots, m$ are known matrices.

We suppose the system states of the PV-HMJSs (9) be available, we design the following $h_{2}(t)$-dependent asynchronous controller:

$$
u(t)=K_{h_{2}(t)}^{p(t)} x(t), \quad h_{2}(t) \in \mathscr{M},
$$

where $K_{h_{2}(t)}^{p(t)}$ is the $h_{2}$-dependent controller gain which will be solved in Theorem 3 .

Submitting the $h_{2}(t)$-dependent controller (7) into the PV-HMJSs (5), we can get the following closed-loop PVHMJSs if $h_{1}(t)=\mathscr{I}$ and $h_{2}(t)=\mathscr{K}, \mathscr{I} \in \mathscr{M}, \mathscr{K} \in \mathscr{M}$ :

$$
\left\{\begin{array}{l}
{\left[\begin{array}{l}
\dot{x}(t) \\
y(t)
\end{array}\right]=\left[\begin{array}{lll}
\bar{A}_{\mathscr{I} \mathscr{K}}^{p} & W_{1 \mathscr{I}}^{p} & F_{\mathscr{I}}^{p} \\
\bar{C}_{\mathscr{I} \mathscr{K}}^{p} & W_{2 \mathscr{I}}^{p} & 0
\end{array}\right]\left[\begin{array}{c}
x(t) \\
\omega(t) \\
f(x(t), t)
\end{array}\right],} \\
\mathscr{I}(0)=x_{0} \\
\mathscr{I}(0)=\mathscr{I}_{0}, \\
\mathscr{K}(0)=\mathscr{K},_{0},
\end{array}\right.
$$

where $\bar{A}_{\mathscr{F} \mathscr{K}}^{p}=A_{\mathscr{I}}^{p}+B_{\mathscr{J}}^{p} K_{\mathscr{K}}^{p}$ and $\bar{C}_{\mathscr{I} \mathscr{K}}^{p}=C_{\mathscr{I}}^{p}+D_{\mathscr{J}}^{p} K_{\mathscr{K}}^{p}$.

\subsection{Main Definitions and Lemmas}

Definition 1 (see [32]). Given two positive constants $0<\mathscr{J}_{1}<\mathscr{J}_{2}$, a weighting matrix $R_{\mathscr{I} \mathscr{K}}>0$, and a finite-time interval [ 0 T $]$, the closed-loop PV-HMJSs (8) with $\omega(t) \equiv 0$ is SFTS within $\left(\mathscr{J}_{1} \mathscr{J}_{2} T R_{\mathscr{I} \mathscr{K}}\right)$ if, for any initial condition $x_{0}^{T} R_{\mathscr{F} \mathscr{K}} x_{0} \leq \mathscr{J}_{1}$, we have

$$
\mathbf{E}\left\{x(t)^{T} R_{\mathscr{I} \mathscr{K}} x(t)\right\} \leq \mathscr{J}_{2}, \quad t \in\left[\begin{array}{ll}
0 & T
\end{array}\right] .
$$

Definition 2 (see [32]). Given two positive constants $0<\mathscr{J}_{1}<\mathscr{J}_{2}$, a weighting matrix $R_{\mathscr{Y} \mathscr{K}}>0$, and a finite-time interval $\left[\begin{array}{ll}0 & T\end{array}\right]$, the closed-loop PV-HMJSs (12) is stochastically finite-time bounded (SFTB) within $\left(\begin{array}{llll}\mathscr{J}_{1} & \mathscr{J}_{2} & T & R_{\mathscr{I} \mathscr{K}}\end{array}\right)$ if, for any initial condition $x_{0}^{T} R_{\mathscr{I} \mathscr{K}} x_{0} \leq \mathscr{J}_{1}$, we have $\mathbf{E}\left\{x(t)^{T} R_{\mathscr{F} \mathscr{K}} x(t)\right\} \leq \mathscr{J}_{2}$, $t \in\left[\begin{array}{ll}0 & T\end{array}\right]$.

Definition 3 (see [6]). Under zero initial condition, the $h_{2}(t)$-dependent controller (9) is said to be a SFTB- $H_{\infty}$ controller of the PV-HMJSs (7) if there exists a $h_{2}(t)$-dependent controller gain $K_{\mathscr{K}}^{p}, \mathscr{K} \in \mathscr{M}$ such that the closedloop PV-HMJSs (10) be SFTB and satisfies the following $H_{\infty}$-gain performance index:

$$
\mathbf{E}\left\{\int_{0}^{T} y^{T}(\tau) y(\tau) \mathrm{d} \tau\right\}<\varphi \mathbf{E}\left\{\int_{0}^{T} \omega^{T}(\tau) \omega(\tau) \mathrm{d} \tau\right\} .
$$

Lemma 1 (see [22]). We have $2 E^{T} X Y F \leqslant \beta E^{T} X^{T} X E+$ $\beta^{-1} F^{T} Y^{T} Y F$ for any given proper dimensional matrices $E$ and $F$, positive scalar $\beta>0$, and matrices $X$ and $Y$.

Assumption 1. For any given positive scalars $T$ and $\omega$, the disturbance input $\omega(t)$ is bounded with $\int_{0}^{T} \omega^{T}(\tau) \omega(\tau) \mathrm{d} \tau \leq \bowtie$

Assumption 2. For a given positive scalar $\vartheta>0$, the unknown state-dependent nonlinear function $f(x(t), t)$ satisfies $\|f(x(t), t)\| \leqslant \vartheta\|x(t)\|$.

Remark 2. In this paper, the transient character in a given finite-time interval is considered for the stochastic linear parameter varying hidden HMJSs. For the given hidden HMJSs with initial conditions (or bounded disturbance input), we said such systems is stochastically finite-time stable (SFTS) (or stochastically finite-time bounded (SFTB)) if the systems' state does not exceed a given limit within a finite-time interval. The specific definitions of the SFTS and 
SFTB are shown in Definitions 1 and 2. For more details of the SFTS and SFTB, we can refer to [31-33].

\section{Main Results}

In this section, we will give some sufficient conditions to solve the SFTB- $H_{\infty}$ observation-mode-based asynchronous controller (11) and obtain the $h_{2}(t)$-dependent controller gain $K_{\mathscr{K}}^{p}, \mathscr{K} \in \mathscr{M}$. We aim the closed-loop PV-HMJSs (12) to fulfill the SFTB- $H_{\infty}$ condition under the action of the observation-mode-based asynchronous controller (11).

Theorem 1. Given four positive constants $\omega>0, \mathscr{J}_{1}>0$, $\omega>0$, and $T>0$, the closed-loop PV-HMJSs (12) is SFTB within $\left(\mathscr{J}_{1} \mathscr{J}_{2} \quad T \quad R_{\mathscr{I} \mathscr{K}} \omega\right)$ if there exist positive constants $0<\mathscr{J}_{1}<\mathscr{J}_{2}, \bar{\lambda}_{P_{\mathscr{F} \mathscr{K}}}>0, \underline{\lambda}_{P_{\mathscr{F}}^{\prime}}>0, \varphi>0$, and $H(t)$-dependent positive-definite symmetric matrices $P_{\mathscr{I} \mathscr{K}}$ and $R_{\mathscr{F} \mathscr{K}}$, where $(\mathscr{I}, \mathscr{K}) \in \mathscr{M} \times \mathscr{M}$, such that

$$
\begin{aligned}
& {\left[\begin{array}{cccc}
\Upsilon & P_{\mathscr{I} \mathscr{K}} W_{1 \mathscr{I}}^{p} & F_{\mathscr{I}}^{p \mathrm{~T}} P_{\mathscr{I} \mathscr{K}} & \Theta_{\mathscr{I} \mathscr{K}} \\
* & -\varphi I & 0 & 0 \\
* & * & -\beta^{-1} I & 0 \\
* & * & * & -\Psi_{\mathscr{I} \mathscr{K}}
\end{array}\right]<0,} \\
& \left(\mathscr{J}_{1} \bar{\lambda}_{P_{\mathscr{F} \mathscr{K}}^{\prime}}+\varphi \omega\right) e^{\omega T}<\mathscr{J}_{2} \underline{\lambda}_{P_{\mathscr{F} \mathscr{K}^{\prime}}{ }^{\prime}}
\end{aligned}
$$

where

$$
\begin{aligned}
& \Upsilon=\operatorname{Her}\left(P_{\mathscr{I} \mathscr{K}} \bar{A}_{\mathscr{I} \mathscr{K}}^{p}\right)+\left(r_{(\mathscr{I}, \mathscr{K})(\mathscr{I}, \mathscr{K})}-\omega\right) P_{\mathscr{I} \mathscr{K}}+\beta^{-1} \vartheta I, \\
& P_{\mathscr{I} \mathscr{K}}^{\prime}=R_{\mathscr{I} \mathscr{K}}^{-(1 / 2)} P_{\mathscr{I} \mathscr{K}} R_{\mathscr{I} \mathscr{K}}^{-(1 / 2)} \\
& \bar{\lambda}_{P_{\mathscr{J} \mathscr{K}}^{\prime}}=\max _{(\mathscr{I}, \mathscr{K}) \in \mathscr{R}} \lambda_{\max }\left(P_{\mathscr{F} \mathscr{K}}^{\prime}\right), \\
& \underline{\lambda}_{P_{\mathscr{I} \mathscr{K}}^{\prime}}=\min _{(\mathscr{S}, \mathscr{K}) \in \mathscr{R}} \lambda_{\min }\left(P_{\mathscr{F} \mathscr{K}}^{\prime}\right), \\
& \Psi_{\mathscr{F} \mathscr{K}}=\operatorname{diag}\left\{P_{\nu_{(\mathscr{Y}, \mathscr{K})}^{(1)}}^{-1}, P_{\nu_{(\mathscr{Y}, \mathscr{K})}^{(2)}}^{-1}, \ldots, P_{\nu_{(\mathscr{Y}, \mathscr{K})}^{\left(h_{(\mathscr{K})}\right)}}^{-1}\right\} \text {, } \\
& \Theta_{\mathscr{I} \mathscr{K}}=\left[\sqrt{\mathscr{R}_{(\mathscr{I}, \mathscr{K})} \nu_{(\mathscr{I}, \mathscr{K})}^{(1)}} I, \ldots, \sqrt{\mathscr{R}_{(\mathscr{I}, \mathscr{K})} v_{(\mathscr{I}, \mathscr{K})}^{\left(h_{(\mathscr{K})}\right)}} I\right] .
\end{aligned}
$$

Proof. Select a SLKF candidate as follows:

$$
V\left(x(t), H_{t}, t\right)=x^{\mathrm{T}}(t) P_{\mathscr{F} \mathscr{K}} x(t) .
$$

Recalling to the closed-loop PV-HMJSs (8), we can get the following weak infinitesimal operator of $V\left(x(t), H_{t}, t\right)$ :

$$
\begin{aligned}
\mathfrak{J} V(x(t), H(t), t) & =\lim _{\tau \longrightarrow 0} \frac{1}{\tau}[\mathbf{E}\{V(x(t+\tau), H(t+\tau), t+\tau) \mid x(t), H(t)\}]-V(x(t), H(t), t) \\
& =x^{T}(t) \mathscr{P}_{1} x(t)+\operatorname{Her}\left(x^{T}(t) P_{\mathscr{I} \mathscr{K}} W_{1 \mathscr{J}}^{p} \omega(t)\right)+\operatorname{Her}\left(x^{T}(t) P_{\mathscr{I} \mathscr{K}} F_{\mathscr{J}}^{p} f x(t), t\right),
\end{aligned}
$$

where

$$
\mathscr{P}_{1}=\left[\operatorname{Her}\left(P_{\mathscr{I} \mathscr{K}} \bar{A}_{\mathscr{F} \mathscr{K}}^{p}\right)+\sum_{(\mathscr{F}, \mathscr{L}) \in \mathscr{R}} \mathscr{R}_{(\mathscr{I}, \mathscr{K})(j, l)} P_{\mathscr{J} \mathscr{L}}\right],
$$

in which

$$
\begin{aligned}
\sum_{(\mathscr{F}, \mathscr{L}) \in \mathscr{R}} \mathscr{R}_{(\mathscr{F}, \mathscr{K})(\mathscr{F}, \mathscr{L})} P_{\mathscr{F} \mathscr{L}}= & \sum_{(\mathscr{F}, \mathscr{L}) \mathscr{R}^{\prime}} \mathscr{R}_{(\mathscr{I}, \mathscr{K})(\mathscr{F}, \mathscr{L})} P_{\mathscr{F} \mathscr{L}} \\
& +r_{(\mathscr{F} \mathscr{K})(\mathscr{F} \mathscr{K})} P_{\mathscr{F} \mathscr{K}} \\
= & \Theta_{\mathscr{F} \mathscr{K}} \Psi_{\mathscr{F} \mathscr{K}}^{-1} \Theta_{\mathscr{F} \mathscr{K}}^{\mathrm{T}} \\
& +r_{(\mathscr{I} \mathscr{K})(\mathscr{F} \mathscr{K})} P_{\mathscr{F} \mathscr{K}},
\end{aligned}
$$

with

$$
\begin{aligned}
& \mathscr{R}^{\prime}=\left\{\mathscr{R}_{(\mathscr{F}, \mathscr{K})(\mathscr{F}, \mathscr{L}) \neq 0,(\mathscr{F}, \mathscr{L}) \in \mathscr{R},(\mathscr{F}, \mathscr{K}) \neq(\mathscr{F}, \mathscr{L})}\right\} \\
& =\left\{v_{(\mathscr{I}, \mathscr{K})}^{(1)} r_{(\mathscr{I}, \mathscr{K})}, \ldots, v_{(\mathscr{I}, \mathscr{K})}^{\left(h_{(\mathscr{T})}\right)} \mathscr{R}_{(\mathscr{F}, \mathscr{K})}\right\}, \\
& \Psi_{\mathscr{Y}, \mathscr{K}}=\operatorname{diag}\left\{P_{v_{(\mathscr{Y}, \mathscr{K})}^{(1)}}^{-1}, P_{v_{(\mathscr{Y}, \mathscr{K})}^{(2)}}^{-1}, \ldots, P_{v_{(\mathscr{I}, \mathscr{K})}^{(h, \mathscr{K})}}^{-1}\right\}, \\
& \Theta_{\mathscr{F}, \mathscr{K}}=\left[\sqrt{r_{(\mathscr{F}, \mathscr{K})} v_{(\mathscr{F}, \mathscr{K})}^{(1)}} I, \ldots, \sqrt{r_{(\mathscr{F}, \mathscr{K})} v_{(\mathscr{Y}, \mathscr{K})}^{\left(h_{(\mathscr{H}, \mathscr{K}}\right)}} I\right] .
\end{aligned}
$$

Recalling to Lemma 1 and Assumption 2, we know that $\mathfrak{I} V(x(t), H(t), t)<x^{T}(t) \mathscr{P}_{2} x(t)+\operatorname{Her}\left(x^{T}(t) P_{\mathscr{I} \mathscr{K}} W_{1 i}^{p} \omega(t)\right)$,

where

$$
\begin{aligned}
\mathscr{P}_{2}= & {\left[\operatorname{Her}\left(P_{\mathscr{F} \mathscr{K}} \bar{A}_{\mathscr{F} \mathscr{K}}^{p}\right)+\sum_{(\mathscr{F}, \mathscr{L}) \in r} r_{(\mathscr{I}, \mathscr{K})(\mathscr{F}, \mathscr{L})} P_{\mathscr{J} \mathscr{L}}\right.} \\
& \left.+\beta F_{\mathscr{I}}^{p^{\mathrm{T}}} P_{\mathscr{F} \mathscr{K}} P_{\mathscr{Y} \mathscr{K}} F_{\mathscr{I}}^{p}+\beta^{-1} \vartheta I\right] .
\end{aligned}
$$

From $\mathfrak{I} V(x(t), H(t), t)<0$, it follows that

$$
\left[\begin{array}{c}
x(t) \\
\omega(t)
\end{array}\right]^{\mathrm{T}}\left[\begin{array}{cc}
\psi & P_{\mathscr{I} \mathscr{K}} W_{1 \mathscr{I}}^{p} \\
* & 0
\end{array}\right]\left[\begin{array}{c}
x(t) \\
\omega(t)
\end{array}\right]<0,
$$

where

$$
\begin{aligned}
\psi= & \operatorname{Her}\left(P_{\mathscr{F} \mathscr{K}} \bar{A}_{\mathscr{F} \mathscr{K}}^{p}\right)+\sum_{(\mathscr{F}, \mathscr{L}) \in r} r_{(\mathscr{I}, \mathscr{K})(\mathscr{F}, \mathscr{L})} P_{\mathscr{F} \mathscr{L}} \\
& +\beta F_{\mathscr{I}}^{p \mathrm{~T}} P_{\mathscr{F} \mathscr{K}} P_{\mathscr{F} \mathscr{K}} F_{\mathscr{I}}^{p}+\beta^{-1} \vartheta I .
\end{aligned}
$$

Recalling to inequalities (11) and (18)-(21), we have the following inequality with positive constant $\omega>0$ : 


$$
\mathbf{E}\{\mathfrak{I} V(x(t), H(t), t)\}<\omega V(x(t), H(t), t)+\varphi \omega^{T}(t) \omega(t) .
$$

Then, we have the following relation by integrating both left and right sides of inequality (23) from 0 to $t$ for $\forall t \in\left[\begin{array}{ll}0 & T\end{array}\right]:$

$$
\begin{gathered}
\mathbf{E}\{V(x(t), H(t), t)\}-\mathbf{E}\{V(x(0), H(0))\} \\
<\varphi \int_{0}^{t} \omega^{T}(\tau) \omega(\tau) \mathrm{d} \tau \\
+\omega \int_{0}^{t} \mathbf{E}\{V(x(t), H(t), \tau)\} \mathrm{d} \tau .
\end{gathered}
$$

Thus,

$\mathbf{E}\{V(x(t), H(t), t)\}<\left(\mathscr{J}_{1} \bar{\lambda}_{P_{\mathscr{J}}^{\prime} \mathscr{K}}+\varphi \omega\right)+\omega \int_{0}^{t} \mathbf{E}\{V(x(t), H(t), \tau)\} \mathrm{d} \tau$.

The above inequality can be rewritten as (26) by considering the Gronwall inequality:

$$
\mathbf{E}\{V(x(t), H(t), t)\}<\left(\mathscr{J}_{1} \bar{\lambda}_{P_{\mathscr{J}}^{\prime}}+\varphi \omega\right) e^{\omega t}<\left(\mathscr{J}_{1} \bar{\lambda}_{P_{\mathscr{J}}^{\prime}}+\varphi \omega\right) e^{\omega T} .
$$

From Rayleigh inequality, we know that $\mathbf{E}\{V(x(t), H(t), t)\}>\underline{\lambda}_{P_{\mathscr{F} \mathscr{K}}} \mathbf{E}\left\{x(t)^{T} R_{\mathscr{I} \mathscr{K}} x(t)\right\}$. Then, we have

$$
\mathbf{E}\left\{x(t)^{T} R_{\mathscr{I} \mathscr{K}} x(t)\right\}<\frac{\left(\mathscr{J}_{1} \bar{\lambda}_{P_{\mathscr{I}}^{\prime}+\varphi}+\varphi\right) e^{\omega T}}{\underline{\lambda}_{P_{\mathscr{J}}^{\prime}}} .
$$

Thus, the $\mathbf{E}\left\{x(t)^{T} R_{\mathscr{I} \mathscr{K}} x(t)\right\}<\mathscr{J}_{2}$ for $\forall t \in\left[\begin{array}{ll}0 & T\end{array}\right]$ holds by inequality (12). This completes the proof.

In Theorem 1, we give some sufficient conditions to ensure the SFTB of the closed-loop PV-HMJSs (12). Then, the SFTB- $H_{\infty}$ condition will be given in the following Theorem 2 on the basis of Theorem 1 .

Theorem 2. Given four positive constants $\omega>0, \mathscr{J}_{1}>0$, $T>0$, and $\omega>0$ with $\int_{0}^{t} \omega^{T}(\tau) \omega(\tau) d \tau \leq \omega$, the SFTB- $H_{\infty}$ condition (10) of the closed-loop PV-HMJSs (8) will be satisfied within $\left(\begin{array}{lllll}\mathscr{J}_{1} & \mathscr{J}_{2} & T & R_{\mathscr{F} \mathscr{K}} & \omega\end{array}\right)$ if there exist positive

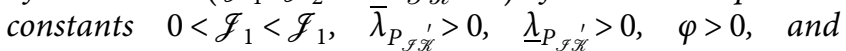
$H(t)$-dependent positive-definite symmetric matrices $P_{\mathscr{I} \mathscr{K}}$ and $R_{\mathscr{I} \mathscr{K}}$, where $(\mathscr{I}, \mathscr{K}) \in \mathscr{M} \times \mathscr{M}$, such that inequalities (12) and (28) hold:

$$
\left[\begin{array}{cccc}
\varkappa & P_{\mathscr{I} \mathscr{K}} W_{1 \mathscr{I}}^{p}+\bar{C}_{\mathscr{I} \mathscr{K}}^{p \mathrm{~T}} W_{2 \mathscr{I}}^{p} & F_{\mathscr{I}}^{p \mathrm{~T}} P_{\mathscr{I} \mathscr{K}} & \Theta_{\mathscr{I} \mathscr{K}} \\
* & W_{2 \mathscr{I}}^{p \mathrm{~T}} W_{2 \mathscr{I}}^{p}-\varphi I & 0 & 0 \\
* & * & -\beta^{-1} I & 0 \\
* & * & * & -\Psi_{\mathscr{I} \mathscr{K}}
\end{array}\right]<0,
$$

where $\mathcal{x}=\Upsilon+e^{\omega T} \bar{C}_{\mathscr{F} \mathscr{K}}^{p T} \bar{C}_{\mathscr{Y} \mathscr{K}}^{p}$.

Proof. The same SLKF is selected as in Theorem 1 and we introduce

$$
\begin{aligned}
\mathbf{E}\{\mathfrak{I} V(x(t), H(t), t)\} & -\omega V(x(t), H(t), t) \\
& <\varphi \omega^{T}(t) \omega(t)-e^{\omega t} \mathbf{E}\left\{y^{T}(t) y(t)\right\} .
\end{aligned}
$$

Inequality (28) guarantees that inequality (29) is established. Then, we use $e^{-\omega t}$ to multiply inequality (29) and integrate such inequality from 0 to $T$ under zero initial condition, and it yields

$$
\left.e^{-\omega t} \mathbf{E}\{V(x(t), H(t), t)\}<\mathbf{E}\left\{\int_{0}^{T}\left(\varphi e^{-\omega \tau} \omega^{T}(\tau) \omega(\tau)-y^{T}(\tau) y(\tau)\right) \mathrm{d} \tau\right)\right\} .
$$

We can rewrite inequality (30) as the following inequality by considering $\mathbf{E}\{V(x(t), H(t), t)\}>0$ :

$$
\begin{aligned}
\mathbf{E}\left\{\int_{0}^{T} y^{T}((\tau) y(\tau)) \mathrm{d} \tau\right\} & <\mathbf{E}\left\{\int_{0}^{T} \varphi e^{-\omega \tau} d^{T}(\tau) \mathrm{d}(\tau) \mathrm{d} \tau\right\} \\
& <\varphi \mathbf{E}\left\{\int_{0}^{T} \omega^{T}(\tau) \omega(\tau) \mathrm{d} \tau\right\} .
\end{aligned}
$$

Thus, we can obtain

$$
\mathbf{E}\left\{\int_{0}^{T} y^{T}((\tau) y(\tau)) \mathrm{d} \tau\right\}<\varphi \mathbf{E}\left\{\int_{0}^{T} \omega^{T}(\tau) \omega(\tau) \mathrm{d} \tau\right\} .
$$

Recalling Definition 3, we can get the SFTB- $H_{\infty}$ condition (10) of the closed-loop PV-HMJSs (8). This completes the proof.

Through the analysis of Theorem 2 , we know that the state feedback controller gain matrix $K_{h_{2}(t)}^{p^{(t)}}$ cannot be solved by Matlab LMI tools due to the nonlinear terms in inequality (28). In the following Theorem 3, some sufficient conditions will be given to obtain finite-time asynchronous controller gain $K_{h_{2}(t)}^{p(t)}$.

Theorem 3. Given four positive constants $\omega>0, \mathscr{J}_{1}>0$, $T>0$, and $\omega>0$ with $\int_{0}^{t} \omega^{T}(\tau) \omega(\tau) d \tau \leq \omega$, there exist a finitetime $H_{\infty}$-gain asynchronous controller with $h_{2}$-dependent state feedback gain $K_{\mathscr{K}}^{p}=Z_{\mathscr{K}}^{p} S_{\mathscr{K}}^{-1}$ such that the SFTB- $H_{\infty}$ condition (10) of the closed-loop PV-HMJSs (12) will be satisfied within $\left(\mathscr{J}_{1} \mathscr{J}_{2} T R_{\mathscr{F} \mathscr{K}} \omega\right)$ if there exist positive scalars $0<\mathscr{J}_{1}<\mathscr{J}_{2}, \underline{\lambda}_{1}>0, \varphi>0, \quad \beta>0, \mu_{\mathscr{I}}>0, \varphi>0$, $H(t)$-dependent positive-definite symmetric matrices $X_{\mathscr{I} \mathscr{K}}$, $R_{\mathscr{I} \mathscr{K}}$, where $\mathscr{I} \in \mathscr{M}, \mathscr{K} \in \mathscr{M}, h_{2}$-dependent positive-definite symmetric matrix $S_{\mathscr{K}}$, and $h_{2}$-dependent matrix $Z_{\mathscr{K}}^{p}$, such that

$$
\begin{gathered}
\mathscr{L}_{1 \mathscr{I} \mathscr{K}}^{p}+\mathscr{B}_{1 \mathscr{I} \mathscr{K}}<0, \\
\underline{\lambda}_{1} R_{\mathscr{I} \mathscr{K}}^{-1}<X_{\mathscr{I} \mathscr{K}}<R_{\mathscr{I} \mathscr{K}}^{-1}, \\
{\left[\begin{array}{cc}
\varphi \omega-\frac{\mathscr{J}_{2} e^{-\omega T}}{\bar{\lambda}_{1}} & \sqrt{\mathscr{J}_{1}} \\
\sqrt{\mathscr{J}_{1}} & -\underline{\lambda}_{1}
\end{array}\right]<0,}
\end{gathered}
$$

where 


$$
\begin{aligned}
& \mathscr{L}_{1 \mathscr{F} \mathscr{K}}^{p}=\operatorname{Her}\left(\left[\begin{array}{c}
A_{\mathscr{I}}^{p} L_{\mathscr{I}} S_{\mathscr{K}}+B_{\mathscr{I}}^{p} Z_{\mathscr{K}}^{p} \\
0 \\
0 \\
\left(C_{\mathscr{I}}^{p} L_{\mathscr{I}} S_{\mathscr{K}}+D_{\mathscr{J}}^{p} Z_{\mathscr{K}}^{p}\right)^{T} \\
e^{\omega T}\left(C_{\mathscr{I}}^{p} L_{\mathscr{I}} S_{\mathscr{K}}+D_{\mathscr{I}}^{p} Z_{\mathscr{K}}^{p}\right)^{T} \\
0
\end{array}\right]\left[\begin{array}{c}
\mu_{\mathscr{J}} I \\
0 \\
0 \\
0 \\
0 \\
0
\end{array}\right]^{T}\right) \text {, } \\
& \mathscr{B}_{1 \mathscr{I} \mathscr{K}}=\left[\begin{array}{cc}
\mathscr{B}_{1 \mathscr{I} \mathscr{K}}^{\prime} & \mathscr{B}_{2 \mathscr{T} \mathscr{K}}^{\prime} \\
* & \mathscr{R}_{3 \mathscr{F} \mathscr{K}}^{\prime}
\end{array}\right] \text {, } \\
& \mathscr{B}_{1 \mathscr{I} \mathscr{K}}^{\prime}=\left[\begin{array}{ccc}
\mathcal{O}_{1 \mathscr{I} \mathscr{K}} & W_{1 \mathscr{I}}^{p} X_{\mathscr{I} \mathscr{K}} & X_{\mathscr{I} \mathscr{K}} F_{\mathscr{I}}^{p \mathrm{~T}} \\
* & \Xi_{5 \mathscr{I}} & 0 \\
* & * & -\beta^{-1} I
\end{array}\right] \text {, } \\
& \mathscr{B}_{2 \mathscr{F} \mathscr{K}}^{\prime}=\left[\begin{array}{ccc}
0 & 0 & X_{\mathscr{I} \mathscr{K}} \Theta_{\mathscr{I} \mathscr{K}} \\
0 & X_{\mathscr{Y} \mathscr{K}} W_{2 \mathscr{I}}^{p \mathrm{~T}} & 0 \\
0 & 0 & 0
\end{array}\right] \text {, } \\
& \mathscr{R}_{3 \mathscr{F} \mathscr{K}}^{\prime}=\operatorname{diag}\left\{-\xi^{-1} I,-\xi I,-\Psi_{\mathscr{Y} \mathscr{K}}^{\prime}\right\} \text {, } \\
& \mathcal{O}_{1 \mathscr{I} \mathscr{K}}=\left(\mathscr{R}_{(i, k)(\mathscr{I}, \mathscr{K})}-\omega\right) X_{\mathscr{I} \mathscr{K}}+\beta^{-1} \vartheta_{\mathscr{Y} \mathscr{K}}, \\
& \Xi_{5 \mathscr{I}}=W_{2 \mathscr{I}}^{p \mathrm{~T}} W_{2 \mathscr{I}}^{p}-\varphi I, \\
& \Theta_{\mathscr{I} \mathscr{K}}=\left[\sqrt{r_{(\mathscr{I}, \mathscr{K})} \nu_{(\mathscr{I}, \mathscr{K})}^{(1)}} I, \ldots, \sqrt{r_{(\mathscr{I}, \mathscr{K})} \nu_{(\mathscr{I}, \mathscr{K})}^{\left(h_{(\mathscr{T})}\right)}} I\right], \\
& \Psi_{\mathscr{Y} \mathscr{K}}^{\prime}=\operatorname{diag}\left\{X_{\mathscr{R}_{(\mathscr{F}, \mathscr{K})}^{(1)}}^{-1}, X_{\mathscr{R}_{(\mathscr{Y}, \mathscr{K})}^{(2)}}^{-1}, \ldots, X_{\mathscr{R}_{(\mathscr{F}, \mathscr{K})}^{(h, \mathscr{K})}}^{-1}\right\} \text {. }
\end{aligned}
$$

Proof. We substitute $\bar{A}_{\mathscr{Y} \mathscr{K}}^{p}$ and $\bar{C}_{\mathscr{I} \mathscr{K}}^{p}$ into inequality (34) and yields

$$
\left[\begin{array}{cccc}
\Xi_{1 \mathscr{I} \mathscr{K}}^{\prime} & \Xi_{4 \mathscr{I} \mathscr{K}} & F_{\mathscr{I}}^{p \mathrm{~T}} P_{\mathscr{F} \mathscr{K}} & \Theta_{\mathscr{J} \mathscr{K}} \\
* & \Xi_{5 \mathscr{I}} & 0 & 0 \\
* & * & -\beta^{-1} I & 0 \\
* & * & * & -\Psi_{\mathscr{J} \mathscr{K}}
\end{array}\right]<0
$$

where

$$
\begin{aligned}
\Xi_{1 \mathscr{I} \mathscr{K}}^{\prime} & =\Xi_{1 \mathscr{I} \mathscr{K}}+\Xi_{2 \mathscr{I} \mathscr{K}}+\Xi_{3 \mathscr{I} \mathscr{K}}, \\
\Xi_{1 \mathscr{I} \mathscr{K}} & =\operatorname{Her}\left(P_{\mathscr{I} \mathscr{K}}\left(A_{\mathscr{I}}^{p}+B_{\mathscr{I}}^{p} K_{\mathscr{K}}^{p}\right)\right), \\
\Xi_{2 \mathscr{I} \mathscr{K}} & =e^{\omega T}\left(C_{\mathscr{I}}^{p}+D_{\mathscr{I}}^{p} K_{\mathscr{K}}^{p}\right)^{T}\left(C_{\mathscr{I}}^{p}+D_{\mathscr{I}}^{p} K_{\mathscr{K}}^{p}\right), \\
\Xi_{3 \mathscr{I} \mathscr{K}} & =\left(\mathscr{R}_{(\mathscr{I}, \mathscr{K})(\mathscr{I} \mathscr{K})}-\omega\right) P_{\mathscr{I} \mathscr{K}}+\beta^{-1} \vartheta I, \\
\Xi_{4 \mathscr{I} \mathscr{K}} & =P_{\mathscr{I} \mathscr{K}} W_{1 \mathscr{I}}^{p}+\left(C_{\mathscr{I}}^{p}+D_{\mathscr{I}}^{p} K_{\mathscr{K}}^{p}\right)^{\mathrm{T}} W_{2 \mathscr{I}}^{p}, \\
\Xi_{5 \mathscr{I}} & =W_{2 \mathscr{I}}^{p \mathrm{~T}} W_{2 \mathscr{I}}^{p}-\varphi I^{p}
\end{aligned}
$$

We use $\operatorname{diag}\left\{P_{\mathscr{F} \mathscr{K}}^{-1}, I, I I\right\}$ to pre- and postmultiply inequality (37), and let $X_{\mathscr{I} \mathscr{K}}=P_{\mathscr{I} \mathscr{K}}^{-1}, Y_{\mathscr{I} \mathscr{K}}^{p}=K_{\mathscr{K}}^{p} X_{\mathscr{I} \mathscr{K}}$, and $Q_{\mathscr{I} \mathscr{K}}=X_{\mathscr{I} \mathscr{K}} X_{\mathscr{I} \mathscr{K}}$, and we use the Schur complement lemma. It can be seen that the following inequality satisfies with positive scalar $\xi>0$ :

$$
\left[\begin{array}{cc}
\mathscr{R}_{1 \mathscr{I} \mathscr{K}}^{\prime} & \mathscr{R}_{2 \mathscr{I} \mathscr{K}}^{\prime} \\
* & \mathscr{R}_{3 \mathscr{F} \mathscr{K}}^{\prime}
\end{array}\right]<0,
$$

where

$$
\begin{aligned}
& \mathscr{R}_{1 \mathscr{I} \mathscr{K}}^{\prime}=\left[\begin{array}{ccc}
\Xi_{1 \mathscr{F} \mathscr{K}}^{\prime} & W_{1 \mathscr{I}}^{p} X_{\mathscr{F} \mathscr{K}} & X_{\mathscr{F} \mathscr{K}} F_{\mathscr{I}}^{p T} \\
* & \Xi_{5 i} & 0 \\
* & * & -\beta^{-1} I
\end{array}\right], \\
& \mathscr{R}_{2 \mathscr{F} \mathscr{K}}^{\prime}=\left[\begin{array}{ccc}
\Xi_{2 \mathscr{I} \mathscr{K}}^{\prime} & \Xi_{3 \mathscr{F} \mathscr{K}}^{\prime} & X_{\mathscr{F} \mathscr{K}} \Theta_{\mathscr{F} \mathscr{K}} \\
0 & X_{\mathscr{F} \mathscr{K}} W_{2 \mathscr{I}}^{p \mathrm{~T}} & 0 \\
0 & 0 & 0
\end{array}\right] \text {, } \\
& \mathscr{R}_{3 \mathscr{I} \mathscr{K}}^{\prime}=\operatorname{diag}\left\{-\xi^{-1},-\xi,-\Psi_{\mathscr{F} \mathscr{K}}\right\} \text {, } \\
& \Psi_{\mathscr{F} \mathscr{K}}^{\prime}=\operatorname{diag}\left\{X_{\mathscr{R}_{(\mathscr{Y}, \mathscr{K})}^{(1)}}^{-1}, X_{\mathscr{R}_{(\mathscr{T}, \mathscr{K})}^{(2)}}^{-1}, \ldots, X_{\mathscr{R}_{(\mathscr{T}, \mathscr{K})}^{\left(h_{(\mathscr{T})}\right)}}^{-1}\right\} \text {, } \\
& \Xi_{1 \mathscr{F} \mathscr{K}}^{\prime}=\operatorname{Her}\left(A_{\mathscr{J}}^{p} X_{\mathscr{J} \mathscr{K}}+B_{\mathscr{J}}^{p} Y_{\mathscr{J} \mathscr{K}}^{p}\right)+\left(r_{(\mathscr{F}, \mathscr{K})(\mathscr{T}, \mathscr{K})}-\omega\right) X_{\mathscr{J} \mathscr{K}} \\
& +\beta^{-1} \vartheta Q_{\mathscr{F} \mathscr{K}}, \\
& \Xi_{2 \mathscr{I} \mathscr{K}}^{\prime}=\left(C_{\mathscr{J}}^{p} X_{\mathscr{F} \mathscr{K}}+D_{\mathscr{J}}^{p} Y_{\mathscr{F} \mathscr{K}}^{p}\right)^{\mathrm{T}} \\
& \Xi_{3 \mathscr{F} \mathscr{K}}^{\prime}=e^{\omega T}\left(C_{\mathscr{I}}^{p} X_{\mathscr{I} \mathscr{K}}+D_{\mathscr{I}}^{p} Y_{\mathscr{I} \mathscr{K}}^{p}\right)^{\mathrm{T}}
\end{aligned}
$$


Considering the asynchronous of (39), we rewrite inequality (39) as follows:

$$
\mathscr{B}_{1 \mathscr{I} \mathscr{K}}+\mathscr{B}_{2 \mathscr{I} \mathscr{K}}<0,
$$

where

$$
\begin{aligned}
& \mathscr{B}_{1 \mathscr{F} \mathscr{K}}=\left[\begin{array}{crr}
\mathscr{B}_{1 \mathscr{F} \mathscr{K}} & \mathscr{B}_{2 \mathscr{F} \mathscr{K}} \\
* & \mathscr{R}_{3 \mathscr{F} \mathscr{K}}
\end{array}\right], \\
& \mathscr{B}_{1 i k}^{\prime}=\left[\begin{array}{ccc}
\mathcal{O}_{1 \mathscr{I} \mathscr{K}} & W_{1 \mathscr{I}}^{p} X_{\mathscr{I} \mathscr{K}} & X_{\mathscr{I} \mathscr{K}} F_{\mathscr{I}}^{p \mathrm{~T}} \\
* & \Xi_{5 \mathscr{I}} & 0 \\
* & * & -\beta^{-1} I
\end{array}\right] \text {, } \\
& \mathscr{B}_{2 \mathscr{F}_{\mathscr{K}}^{\prime}}^{\prime}=\left[\begin{array}{ccc}
0 & 0 & X_{\mathscr{I} \mathscr{K}} \Theta_{\mathscr{F} \mathscr{K}} \\
0 & X_{\mathscr{I} \mathscr{K}} W_{2 \mathscr{I}}^{p \mathrm{~T}} & 0 \\
0 & 0 & 0
\end{array}\right] \text {, } \\
& \mathscr{B}_{2 \mathscr{F} \mathscr{K}}=\operatorname{Her}\left(\left[\begin{array}{c}
A_{\mathscr{I}}^{p} X_{\mathscr{I} \mathscr{K}}+B_{\mathscr{I}}^{p} Y_{\mathscr{I} \mathscr{K}}^{p} \\
0 \\
0 \\
\left(C_{\mathscr{J}}^{p} X_{\mathscr{I} \mathscr{K}}+D_{\mathscr{I}}^{p} Y_{\mathscr{I} \mathscr{K}}^{p}\right)^{T} \\
e^{\omega T}\left(C_{\mathscr{I}}^{p} X_{\mathscr{I} \mathscr{K}}+D_{\mathscr{I}}^{p} Y_{\mathscr{I} \mathscr{K}}^{p}\right)^{T} \\
0
\end{array}\right]\left[\begin{array}{l}
I \\
0 \\
0 \\
0 \\
0 \\
0
\end{array}\right]^{T}\right) \\
& \mathcal{O}_{1 \mathscr{I} \mathscr{K}}=\left(r_{(\mathscr{I}, \mathscr{K})(\mathscr{I}, \mathscr{K})}-\omega\right) X_{\mathscr{I} \mathscr{K}}+\beta^{-1} \vartheta Q_{\mathscr{I} \mathscr{K}}
\end{aligned}
$$

For $\mathscr{B}_{2 \mathscr{I} \mathscr{K}}$, we define $X_{\mathscr{I} \mathscr{K}}=\mu_{\mathscr{I}} L_{\mathscr{J}} S_{\mathscr{K}}$, where $L_{\mathscr{I}}$ is a nonsingular unit matrix for $\forall i \in \mathscr{M}$. We can obtain

$$
\left\{\begin{array}{l}
A_{\mathscr{I}}^{p} X_{\mathscr{I} \mathscr{K}}+B_{\mathscr{J}}^{p} Y_{\mathscr{I} \mathscr{K}}^{p}=\mu_{\mathscr{I}}\left(A_{\mathscr{I}}^{p}+B_{\mathscr{I}}^{p} K_{\mathscr{K}}^{p}\right) L_{\mathscr{I}} S_{\mathscr{K}} \\
C_{\mathscr{I}}^{p} X_{\mathscr{I} \mathscr{K}}+D_{\mathscr{I}}^{p} Y_{\mathscr{I} \mathscr{K}}^{p}=\mu_{\mathscr{I}}\left(C_{\mathscr{I}}^{p}+D_{\mathscr{I}}^{p} K_{\mathscr{K}}^{p}\right) L_{\mathscr{I}} S_{\mathscr{K}} .
\end{array}\right.
$$

Since $L_{\mathscr{I}}$ is a nonsingular unit matrix, we can get inequality (41) by inequality (33) with $Z_{\mathscr{I} \mathscr{K}}^{p}=K_{\mathscr{K}}^{p} L_{\mathscr{I}} S_{\mathscr{K}}$. Moreover, we know that $\operatorname{Her}\left(L_{\mathscr{J}} S_{\mathscr{K}}\right)>0$, i.e., $\operatorname{Her}\left(S_{\mathscr{K}}\right)>0$ from inequality (33). Thus, $Z_{\mathscr{K}}^{p}$ is also a nonsingular matrix, which means that $K_{\mathscr{K}}^{p}=Z_{\mathscr{K}}^{p} S_{\mathscr{K}}^{-1}$ can be defined and solved.

Defining $\bar{X}_{\mathscr{Y} \mathscr{K}}=R_{\mathscr{I} \mathscr{K}}^{-(1 / 2)} X_{\mathscr{I} \mathscr{K}} R_{\mathscr{I} \mathscr{K}}^{-(1 / 2)}, \bar{\lambda}_{\bar{X}_{\mathscr{I}}}=\max _{(\mathscr{I}, \mathscr{K}) \epsilon r}$ $\lambda_{\text {max }}\left(\bar{X}_{\mathscr{F} \mathscr{K}}\right)$, and $\underline{\lambda}_{\bar{X}_{\mathscr{Y} \mathscr{K}}}=\min _{(\mathscr{I}, \mathscr{K}) \in r} \lambda_{\min }\left(\bar{X}_{\mathscr{I} \mathscr{K}}\right)$, letting $\underline{\lambda}_{1} \leq \underline{\lambda}_{\bar{X}_{\mathscr{I}}}$ and $\bar{\lambda}_{1} \geq \bar{\lambda}_{\bar{X}_{\mathscr{I} \mathscr{K}}}$, and considering $\bar{\lambda}_{\bar{X}_{\mathscr{I}}}=1 / \underline{\lambda}_{P_{\mathscr{S}}^{\prime} \text { and }}$ $\underline{\lambda}_{\bar{X}_{\mathscr{S}}}=1 / \bar{\lambda}_{P_{\mathscr{S}}^{\prime}}$, inequality (12) can be rewritten as

$$
\frac{\mathscr{J}_{1}}{\underline{\lambda}_{1}}+\varphi \omega<\frac{\mathscr{J}_{2} e^{-\omega T}}{\bar{\lambda}_{1}}
$$

By considering the eigenvalue conversion method and using the Schur complement lemma, inequalities (34) and (35) can be obtained. This completes the proof.

However, we also cannot get the state feedback controller gain matrix $K_{h(t)}^{p(t)}$ by Matlab LMI tools in Theorem 3 due to the dependence of the parameters. Next, the methods of approximate basis function and gridding technique will be used to deparameterize the parameter-dependent matrices in Theorem 3. The specific deparameterization process is as follows:

$$
\begin{gathered}
\mathscr{A}^{p}=\mathscr{A}_{0}^{p}+\sum_{\mathscr{I}=1}^{m} \mathscr{A}_{\mathscr{I}} p_{\mathscr{I}}<0, \quad \forall p \in \mathcal{Z}, \\
\left.\boldsymbol{Z}=\left\{p(t) \in \mathbb{R}^{p}: \underline{p}_{1 \mathscr{I}} \leqslant p_{\mathscr{I}}(t) \leqslant \bar{p}_{1 \mathscr{I}}, \quad \forall \mathscr{I} \in 1,2, \ldots, m\right]\right\},
\end{gathered}
$$

where $p(t)$ is the time-varying parameter. From inequalities (45) and (46), we know that $\mathcal{Z}$ denotes an LMI and $\mathscr{A}^{P}$ has infinite number of LMIs. Then, we select the following basis function $\left\{F_{\mathscr{I}}^{(p(t))}\right\}_{\mathcal{f}=1}^{n_{f}}$ to solve inequality (45):

$$
\mathscr{A}_{\mathscr{I}}^{p(t)}=\sum_{\mathscr{J}=1}^{n_{f}} F_{\mathscr{I}}^{(p(t))} \mathscr{A}_{\mathscr{I} \mathscr{f}} .
$$

Furthermore, the infinite LMIs of the $\mathscr{A}^{p}$ can be transformed into the finite ones if we divide the space of parameter changes into finite-dimensional grids, which means the following relation satisfied for each set of parameters on the grid for $\forall p(t) \in \mathbb{Z}$ :

$$
\mathscr{A}^{p}=\sum_{\mathscr{J}=1}^{n_{f}} F_{\mathscr{J}}^{(p(t))} \mathscr{A}_{0}+\sum_{\mathcal{J}=1}^{n_{f}} \sum_{i=1}^{m} F_{\mathscr{J}}^{(p(t))} \mathscr{A}_{\mathscr{I} \mathscr{J}}<0 .
$$

Remark 3. In Theorem 3, the difficulty of calculation will be increased because of the asynchronous characteristic between the controller modes and the system modes. In order to solve such difficulty, the controller modes $h_{2}(t)$ is converted $h_{1}(t)$. Moreover, some sufficient conditions are obtained to make the closed-loop HMJSs with linear parameter varying be SFTB- $H_{\infty}$ by introduced auxiliary variables.

Remark 4. In addition, we have introduced the stochastic Lyapunov-Krasovskii functional methods in Theorem 3, which will induce somewhat conservatism of the main results. In future work, we can reduce the impact of conservativeness through replacing the quasi-one-sided Lipschitz condition or one-sided Lipschitz condition with local Lipschitz condition in Assumption 2.

\section{Numerical Example}

In this section, we consider a class of PV-HMJSs with two subsystems.

Subsystem 1: 


$$
\begin{aligned}
& A_{1}^{p(t)}=\left[\begin{array}{cc}
-16.5 & 6 \\
7 & -12.1
\end{array}\right]+\left[\begin{array}{cc}
0.05 & 0 \\
0 & 0.05
\end{array}\right] \sin (t) \\
& +\left[\begin{array}{cc}
0.02 & 0 \\
0 & 0.02
\end{array}\right] \cos (t) \text {, } \\
& B_{1}^{p(t)}=\left[\begin{array}{l}
1 \\
1
\end{array}\right]+\left[\begin{array}{l}
0.2 \sin (t) \\
0.1 \cos (t)
\end{array}\right] \text {, } \\
& W_{11}^{p(t)}=[0.5 \sin (t)+0.2 \cos (t)] \text {, } \\
& F_{1}^{p(t)}=[0.4 \sin (t)+0.1 \cos (t)] \text {, } \\
& W_{21}^{p(t)}=\left[\begin{array}{ll}
0.3 & 0.1 \\
0.2 & 0.2
\end{array}\right]+\left[\begin{array}{cc}
0.01 & 0 \\
0 & 0.01
\end{array}\right] \sin (t) \\
& +\left[\begin{array}{cc}
0.02 & 0 \\
0 & 0.02
\end{array}\right] \cos (t) \text {, } \\
& C_{1}^{p(t)}=\left[\begin{array}{cc}
-3.3 & -0.2 \\
0.1 & -2.8
\end{array}\right]+\left[\begin{array}{cc}
-0.1 & 0 \\
0 & -0.2
\end{array}\right] \sin (t) \\
& +\left[\begin{array}{cc}
-0.3 & 0 \\
0 & -0.2
\end{array}\right] \cos (t) \text {, } \\
& D_{1}^{p(t)}=\left[\begin{array}{l}
0.3 \\
0.2
\end{array}\right]+\left[\begin{array}{c}
0.1 \sin (t) \\
0.02 \cos (t)
\end{array}\right] \text {. }
\end{aligned}
$$

Subsystem 2:

$$
\begin{aligned}
& A_{2}^{p(t)}=\left[\begin{array}{cc}
-12.3 & 8 \\
4 & -11.2
\end{array}\right]+\left[\begin{array}{cc}
0.02 & 0 \\
0 & 0.01
\end{array}\right] \sin (t) \\
& +\left[\begin{array}{cc}
0.01 & 0 \\
0 & 0.01
\end{array}\right] \cos (t) \text {, } \\
& B_{2}^{p(t)}=\left[\begin{array}{l}
2 \\
2
\end{array}\right]+\left[\begin{array}{l}
0.4 \sin (t) \\
0.3 \cos (t)
\end{array}\right] \text {, } \\
& W_{12}^{p(t)}=[0.4 \sin (t)+0.2 \cos (t)] \text {, } \\
& F_{2}^{p(t)}=[0.3 \sin (t)+0.1 \cos (t)] \text {, } \\
& W_{22}^{p(t)}=\left[\begin{array}{ll}
0.1 & 0.2 \\
0.2 & 0.1
\end{array}\right]+\left[\begin{array}{cc}
0.01 & 0 \\
0 & 0.01
\end{array}\right] \sin (t) \\
& +\left[\begin{array}{cc}
0.02 & 0 \\
0 & 0.02
\end{array}\right] \cos (t) \\
& C_{2}^{p(t)}=\left[\begin{array}{cc}
-2.8 & -0.1 \\
0.2 & -3.1
\end{array}\right]+\left[\begin{array}{cc}
-0.2 & 0 \\
0 & -0.2
\end{array}\right] \sin (t) \\
& +\left[\begin{array}{cc}
-0.3 & 0 \\
0 & -0.1
\end{array}\right] \cos (t) \text {, } \\
& D_{2}^{p(t)}=\left[\begin{array}{l}
0.3 \\
0.2
\end{array}\right]+\left[\begin{array}{c}
0.2 \sin (t) \\
0.01 \cos (t)
\end{array}\right] \text {, }
\end{aligned}
$$

where $\sin (t)$ and $\cos (t)$ are time-varying parameters and bounded with $\left[\begin{array}{ll}-1 & 1\end{array}\right]$. We assume the weighted matrix $R=I$, the initial consideration $\mathscr{J}_{1}=0.8$, the external disturbance $\omega(t)=\left[\begin{array}{c}0.1 \sin ^{2}(2 t) \\ 0.2 \sin ^{2}(t)\end{array}\right]$, and the unknown statedependent nonlinear function $f(x(t), t)=\left[\begin{array}{l}0.3 x_{1}(t) \\ 0.1 x_{2}(t)\end{array}\right]$ in finite-time interval $T=5$. The other values of the constants are given as $\omega=0.3, \bar{\lambda}_{1}=5.089, \underline{\lambda}_{1}=0.5, \mu_{1}=1.2, \mu_{2}=1.5$, $\omega=0.3, \beta=0.3, \vartheta=1$, and $\xi=0.2$.

We assume the $h_{2}(t)$-dependent controller gain $K_{\mathscr{K}}^{p}$ with two control schemes for two different jump modes, i.e., $\mathscr{M} \in\{1,2\}$ and $\mathscr{M} \in\{1,2\}$. The $\Pi$-dependent conditional probability matrices are $\aleph^{1}=\left[\begin{array}{cc}-0.6 & 0.6 \\ 0.4 & -0.4\end{array}\right]$ and $\aleph^{2}=\left[\begin{array}{cc}0.3 & -0.3 \\ -0.1 & 0.1\end{array}\right]$. The parameters of $\alpha_{j l}^{k}$ are $\alpha_{1}=\alpha_{j l}^{1}=\left[\begin{array}{ll}0.3 & 0.7 \\ 0.4 & 0.6\end{array}\right]$ and $\alpha_{2}=\alpha_{j l}^{2}=\left[\begin{array}{ll}0.2 & 0.8 \\ 0.5 & 0.5\end{array}\right]$.

Solving LMIs (33)-(35), we can get the following finitetime state feedback gain matrices:

$$
\begin{aligned}
& K_{1}^{p}=\left[\begin{array}{ll}
2.9251 & -1.1431
\end{array}\right]+\left[\begin{array}{ll}
-1.1633 \sin (t) & 0.3333 \cos (t)
\end{array}\right], \\
& K_{2}^{p}=\left[\begin{array}{ll}
3.0817 & -0.7694
\end{array}\right]+\left[\begin{array}{ll}
-0.5792 \sin (t) & -0.171 \cos (t)
\end{array}\right] .
\end{aligned}
$$

Meanwhile, the other relevant solutions are given as $\mathscr{J}_{2}=5.0894$ and $\varphi=9.8$. Then, the simulation results are shown in Figures 1-3.

The jump modes of $h_{1}(t)$ and $h_{2}(t)$ are shown in Figure 1 . The state trajectory $x(t)$ and $y(t)$ of the closedloop PV-HMJSs (8) are shown in Figures 2 and 3. From Figures 2 and 3, we can seen the SFTB- $H_{\infty}$ condition is satisfied with $\mathbf{E}\left\{x(t)^{T} R_{\mathscr{I} \mathscr{K}} x(t)\right\}<\mathscr{J}_{2}$ in which $\mathscr{J}_{2}=5.0894$ and $\varphi=9.8$.

Remark 5. Similar to some results of reinforcement learning methods [41-44], this paper also considered the problem of stabilization of stochastic Markov jump systems. Moreover, we also use the hidden Markov model to denote the asynchronous characteristic between system modes and controller modes. Different from some existing results on the finite-time control problem $[35,36]$, the problem of SFTB- $H_{\infty}$ asynchronous control is studied for continuous-time HMJSs via parameter varying in this paper. Compared with the existing results of asynchronous control for discrete-time HMJSs [37-40], this paper firstly considers the asynchronous stabilization problem for the continuous-time HMJSs with parameter varying models.

For the future work, we can learn from the reinforcement learning methods to study the problem of finite-time asynchronous online control for the continuous-time PVHMJSs. 


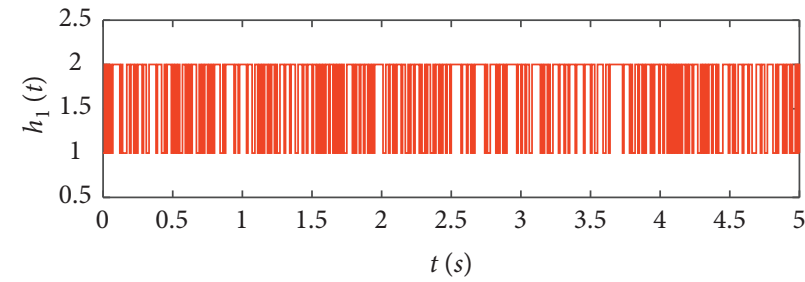

(a)

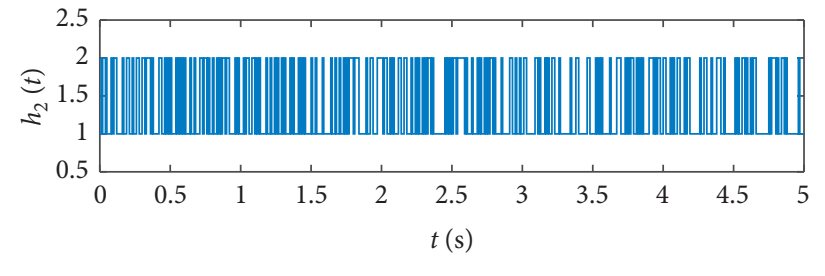

(b)

Figure 1: The jump mode $H(t)$ of the PV-HMJSs. (a) The jump mode of the hidden state $h_{1}(t)$. (b) The jump mode of the observation state $h_{2}(t)$.

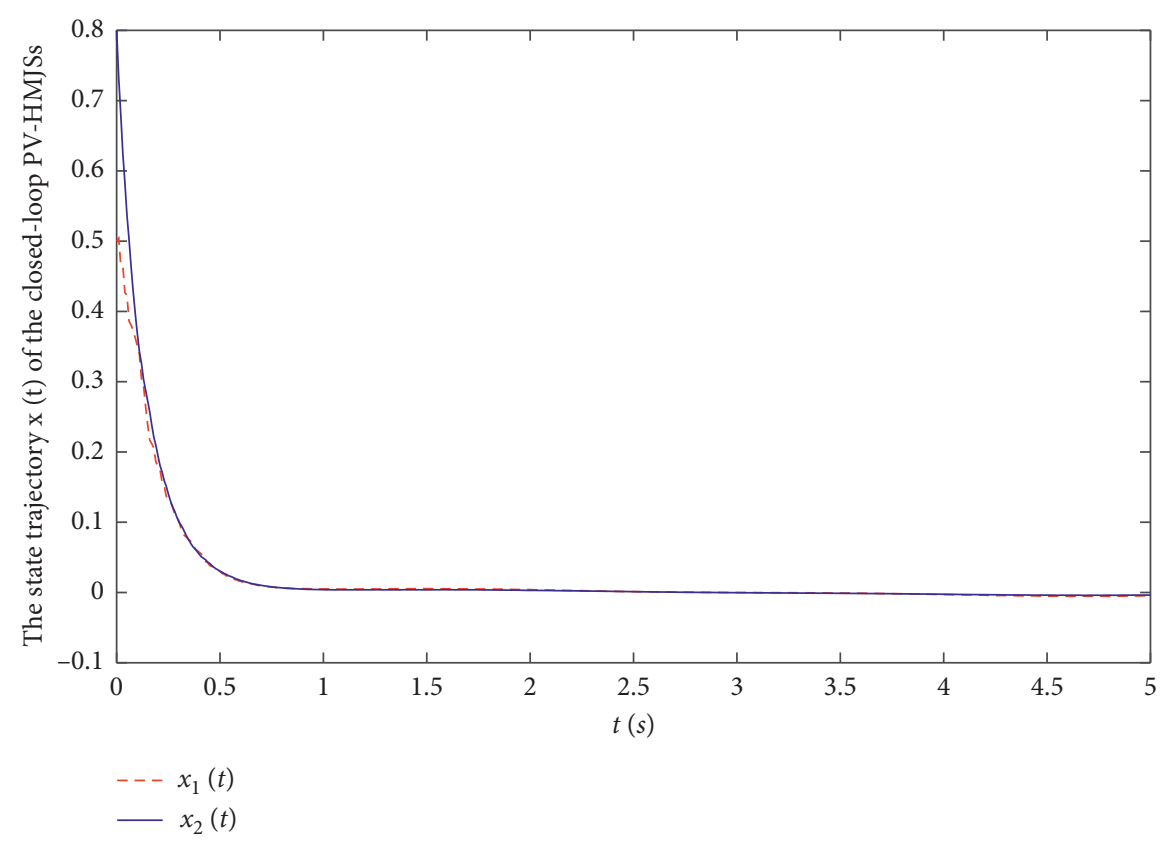

Figure 2: The $x(t)$ of the closed-loop PV-HMJSs.

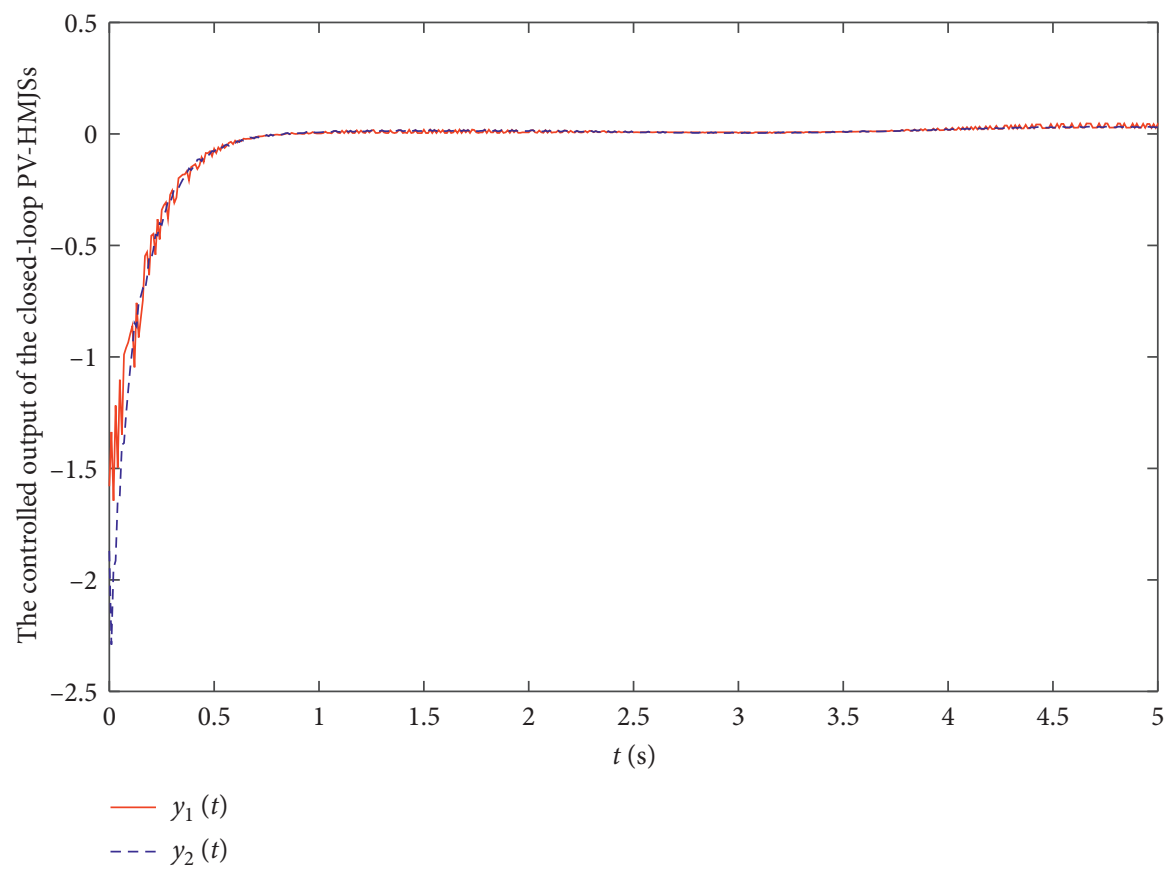

FIgURe 3: The $y(t)$ of the closed-loop PV-HMJSs. 


\section{Conclusion}

This paper studied the SFTB- $H_{\infty}$ asynchronous control problem for continuous-time PV-HMJSs. Some sufficient conditions are given to solve the SFTB- $H_{\infty}$ asynchronous control gain by considering the methods of SLKF and LMIs. The designed SFTB- $H_{\infty}$ asynchronous controller makes the closed-loop PV-HMJSs satisfy the SFTB- $H_{\infty}$ condition. Finally, we use a numerical example to show the validity of the main results of this paper. For the future research work, the asynchronous control problem for fuzzy PV-HMJSs apply approximation method will be considered.

\section{Data Availability}

The data findings of this study are available from the corresponding author upon request.

\section{Conflicts of Interest}

The authors declare that they have no conflicts of interest.

\section{References}

[1] Y. A. Zabala and O. L. V. Costa, "Static output constrained control for discrete-time hidden Markov jump linear systems," IEEE Access, vol. 8, pp. 62969-62979, 2020.

[2] A. M. de Oliveira, O. L. V. Costa, and J. Daafouz, "Design of stabilizing dynamic output feedback controllers for hidden Markov jump linear systems," IEEE Control Systems Letters, vol. 2, no. 2, pp. 278-283, 2018.

[3] P. Cheng, J. Wang, S. He, X. Luan, and F. Liu, "Observerbased asynchronous fault detection for conic-type nonlinear jumping systems and its application to separately excited DC motor," IEEE Transactions on Circuits and Systems I: Regular Papers, vol. 67, no. 3, pp. 951-962, 2020.

[4] F. Li, S. Xu, and B. Zhang, "Resilient asynchronous $H_{\infty}$ control for discrete-time Markov jump singularly perturbed systems based on hidden Markov model," IEEE Transactions on Systems, Man, and Cybernetics: Systems, vol. 50, no. 8, pp. 2420-2430, 2019.

[5] S. Dong, Z.-G. Wu, P. Shi, H. Su, and T. Huang, "Quantized control of Markov jump nonlinear systems based on fuzzy hidden Markov model," IEEE Transactions on Cybernetics, vol. 49, no. 7, pp. 2420-2430, 2019.

[6] C. Ren, S. He, X. Luan, F. Liu, and H. R. Karimi, "Finite-time $L_{2}$-gain asynchronous control for continuous-time positive hidden Markov jump systems via T-S fuzzy model approach," IEEE Transactions on Cybernetics, pp. 9-1, 2020.

[7] F. Stadtmann and O. L. V. Costa, " $\mathrm{H}_{2}$-control of continuoustime hidden Markov jump linear systems," IEEE Transactions on Automatic Control, vol. 62, no. 8, pp. 4031-4037, 2017.

[8] S. Dong, Z.-G. Wu, Y.-J. Pan, H. Su, and Y. Liu, "HiddenMarkov-model-based asynchronous filter design of nonlinear Markov jump systems in continuous-time domain," IEEE Transactions on Cybernetics, vol. 49, no. 6, pp. 2294-2304, 2019.

[9] F. Stadtmann and O. L. V. Costa, "Exponential hidden Markov models for $H_{\infty}$ control of jumping systems," IEEE Control Systems Letters, vol. 2, no. 4, pp. 845-850, 2018.

[10] J. Cheng, C. K. Ahn, H. R. Karimi, J. Cao, and W. Qi, “An event-based asynchronous approach to Markov jump systems with hidden mode detections and missing measurements,"
IEEE Transactions on Systems, Man, and Cybernetics: Systems, vol. 49, no. 9, pp. 1749-1758, 2019.

[11] H. Zheng, D. Yang, X. Xie, and Z. Zhang, "An adaptive algorithm for active vibration control of parameter-varying systems with a new online secondary path estimation method," IEEE Signal Processing Letters, vol. 27, pp. 705-709, 2020.

[12] M. S. de Oliveira and R. L. Pereira, "LMI-based filter design conditions for discrete-time LPV systems with bounded parameter variation," IEEE Transactions on Automatic Control, 2019.

[13] M. M. Seron and J. A. De Doná, "Robust fault estimation and compensation for LPV systems under actuator and sensor faults," Automatica, vol. 52, pp. 294-301, 2015.

[14] D. Rotondo, F. Nejjari, V. Puig, and J. Blesa, "Model reference FTC for LPV systems using virtual actuators and set-membership fault estimation," International Journal of Robust and Nonlinear Control, vol. 25, no. 5, pp. 735-760, 2015.

[15] W. M. H. Heemels, J. Daafouz, and G. Millerioux, "Observerbased control of discrete-time LPV systems with uncertain parameters," IEEE Transactions on Automatic Control, vol. 55, no. 9, pp. 2130-2135, 2010.

[16] M. Fleps-Dezasse, F. Svaricek, and J. Brembeck, "Design and experimental assessment of an active fault-tolerant LPV vertical dynamics controller," IEEE Transactions on Control Systems Technology, vol. 27, no. 3, pp. 1267-1274, 2019.

[17] J. Theis, P. Seiler, and H. Werner, "LPV model order reduction by parameter-varying oblique projection," IEEE Transactions on Control Systems Technology, vol. 26, no. 3, pp. 773-784, 2018.

[18] P. B. Cox, S. Weiland, and R. Tóth, "Affine parameter-dependent Lyapunov functions for LPV systems with affine dependence," IEEE Transactions on Automatic Control, vol. 63, no. 11, pp. 3865-3872, 2018.

[19] W. Qi, G. Zong, and H. R. Karimi, " $H_{\infty}$ control for positive delay systems with semi-Markov process and application to a communication network model," IEEE Transactions on Industrial Electronics, vol. 66, no. 3, pp. 2081-2091, 2019.

[20] J. Zhao, F. Gao, L. Kuang, Q. Wu, and W. Jia, "Channel tracking with flight control system for UAV mmWave MIMO communications," IEEE Communications Letters, vol. 22, no. 6, pp. 1224-1227, 2018.

[21] B. Chen, Y. Yang, B. Lee, and T. Leer, "Fuzzy adaptive predictive flow control of ATM network traffic," IEEE Transactions on Fuzzy Systems, vol. 11, no. 4, pp. 568-581, 2003.

[22] J. Xia, J. Zhang, W. Sun, B. Zhang, and Z. Wang, "Finite-time adaptive fuzzy control for nonlinear systems with full state constraints," IEEE Transactions on Systems, Man, and Cybernetics: Systems, vol. 49, no. 7, pp. 1541-1548, 2019.

[23] H. Wang, P. X. Liu, X. Zhao, and X. Liu, "Adaptive fuzzy finite-time control of nonlinear systems with actuator faults," IEEE Transactions on Cybernetics, vol. 50, no. 5, pp. 17861797, 2020.

[24] N. Wang, H. R. Karimi, H. Li, and S. Su, "Accurate trajectory tracking of disturbed surface vehicles: a finite-time control approach," IEEE/ASME Transactions on Mechatronics, vol. 24, no. 3, pp. 1064-1074, 2019.

[25] H. Ren, G. Zong, and T. Li, "Event-triggered finite-time control for networked switched linear systems with asynchronous switching," IEEE Transactions on Systems, Man, and Cybernetics: Systems, vol. 48, no. 11, pp. 1874-1884, 2018.

[26] P. Du, H. Liang, S. Zhao, and C. K. Ahn, "Neural-based decentralized adaptive finite-time control for nonlinear largescale systems with time-varying output constraints," IEEE 
Transactions on Systems, Man, and Cybernetics: Systems, pp. 1-12, 2019.

[27] F. Wang and X. Zhang, "Adaptive finite time control of nonlinear systems under time-varying actuator failures," IEEE Transactions on Systems, Man, and Cybernetics: Systems, vol. 49, no. 9, pp. 1845-1852, 2019.

[28] B. Xu, "Composite learning finite-time control with application to quadrotors," IEEE Transactions on Systems, Man, and Cybernetics: Systems, vol. 48, no. 10, pp. 1806-1815, 2018.

[29] H. Du, G. Wen, Y. Cheng, and J. Lu, "Design and implementation of bounded finite-time control algorithm for speed regulation of permanent magnet synchronous motor," IEEE Transactions on Industrial Electronics, 2020.

[30] S. Li, C. K. Ahn, and Z. Xiang, "Command filter based adaptive fuzzy finite-time control for switched nonlinear systems using state-dependent switching method," IEEE Transactions on Fuzzy Systems, 2019.

[31] S. Zheng and W. Li, "Fuzzy finite time control for switched systems via adding a barrier power integrator," IEEE Transactions on Cybernetics, vol. 49, no. 7, pp. 2693-2706, 2019.

[32] C. Ren and S. He, "Finite-time stabilization for positive Markovian jumping neural networks," Applied Mathematics and Computation, vol. 365, Article ID 124631, 2020.

[33] C. Ren, R. Nie, and S. He, "Finite-time positiveness and distributed control of Lipschitz nonlinear multi-agent systems," Journal of the Franklin Institute, vol. 356, no. 15, pp. 8080-8092, 2019.

[34] C. Ren and S. He, "Sliding mode control for a class of nonlinear positive Markov jumping systems with uncertainties in a finite-time interval," International Journal of Control, Automation and Systems, vol. 17, no. 7, pp. 16341641, 2019.

[35] P. Cheng, S. He, J. Cheng, X. Luan, and F. Liu, “Asynchronous output feedback control for a class of conic-type nonlinear hidden Markov jump systems within a finite-time interval," IEEE Transactions on Systems, Man, and Cybernetics, 2020.

[36] P. Cheng and S. He, "Observer-based finite-time asynchronous control for a class of hidden Markov jumping systems with conic-type non-linearities," IET Control Theory and Applications, vol. 14, no. 2, pp. 244-252, 2020.

[37] J. Song, Y. Niu, and Y. Zou, "Asynchronous sliding mode control of Markovian jump systems with time-varying delays and partly accessible mode detection probabilities," Automatica, vol. 93, pp. 33-41, 2018.

[38] J. Song, Y. Niu, and Y. Zou, “Asynchronous output feedback control of time-varying Markovian jump systems within a finite-time interval," Journal of the Franklin Institute, vol. 354, no. 15, pp. 6747-6765, 2017.

[39] M. Fang, L. Wang, and Z. Wu, "Asynchronous stabilization of boolean control networks with stochastic switched signals," IEEE Transactions on Systems, Man, and Cybernetics: Systems, pp. 1-8, 2019.

[40] S. Dong, M. Fang, P. Shi, Z. Wu, and D. Zhang, "Dissipativitybased control for fuzzy systems with asynchronous modes and intermittent measurements," IEEE Transactions on Cybernetics, vol. 50, no. 6, pp. 2389-2399, 2018.

[41] S. He, H. Fang, M. Zhang, F. Liu, and Z. Ding, "Adaptive optimal control for a class of nonlinear systems: the online policy iteration approach," IEEE Transactions on Neural Networks and Learning Systems, vol. 31, no. 2, pp. 549-558, 2020.

[42] S. He, H. Fang, M. Zhang, F. Liu, X. Luan, and Z. Ding, "Online policy iterative-based $H_{\infty}$ optimization algorithm for a class of nonlinear systems," Information Sciences, vol. 495, pp. 1-13, 2019.

[43] S. He, H. Fang, M. Zhang, F. Liu, X. Luan, and Z. Ding, "Reinforcement learning and adaptive optimization of a class of Markov jump systems with completely unknown dynamic information," Neural Computing and Applications, vol. 32, pp. 14311-14320, 2020.

[44] C. Wang, H. Fang, and S. He, "Adaptive optimal controller design for a class of LDI-based neural network systems with input time-delays," Neurocomputing, vol. 385, pp. 292-299, 2020. 